\section{Rapporto e decisioni prese alla Camera medica straordinaria del 2 febbraio 2000}

\author{
F.-X. Deschenaux
}

Questa seduta straordinaria della Camera medica svizzera è stata dedicata esclusivamente alla discussione delle proposte concernenti la struttura tariffaria TarMed, la tariffa medica AINF/AM/AI, come pure la procedura decisionale per l'introduzione della suddetta struttura nelle tariffe cantonali della LAMal e alle decisioni in merito.

La seduta viene aperta alle ore 9.40 e subito si costata che il quorum è stato raggiunto. Il presidente, il dott. H. H. Brunner, informa l'assemblea che due invitati assisteranno al dibattito. Si tratta del dott. M. Ganz, presidente dell'Associazione svizzera dei medici indipendenti che lavorano in cliniche private (ASMI/SVBP), come anche dell'avvocato F. Bernath, segretario generale della Foederatio Medicorum Scrutantium (FMS). Il presidente ottiene tacitamente dalla Camera il permesso per questi due invitati di prendere la parola. Si dichiara inoltre che alcune proposte sono giunte dopo il termine che era stato fissato e che non è più stato possibile farle figurare nella documentazione consegnata ai delegati; le discussioni con gli autori di queste proposte hanno permesso di costatare che o concordavano con altre proposte pervenute entro i termini oppure che non ponevano problemi e potevano essere integrate nella discussione. Il presidente preannuncia anche ai delegati che le operazioni di voto sulla struttura tariffaria alfa 2.2 avranno luogo al più tardi alle ore 15.30 , qualunque sia lo stadio raggiunto dalle deliberazioni.

Dopo aver designato sei scrutatrici e scrutatori, si è entrati in materia.

\section{Condizioni statutarie per un'eventuale votazione generale}

Il presidente dichiara che i delegati sono stati informati per iscritto sulla composizione di questa Camera medica straordinaria come anche su un'eventuale votazione generale e, poiché nessuno chiede la parola su questo oggetto, costata che queste spiegazioni scritte non sono contestate e che possono quindi essere considerate tacitamente accettate.

\section{Riassunto della situazione delle negoziazioni e della situazione politica}

Il presidente ricorda che il secondo semestre del 1999 è stato utilizzato essenzialmente per la trasposizione del documento beta 3 nelle versioni "alfa" della struttura tariffaria (oggi la versione «alfa 2.2»). È durante questi lavori di trasposizione che sono state prese in considerazione alcune proposte del Sorvegliante dei prezzi dai partner delle negoziazioni. Questi lavori hanno mobilizzato importanti risorse di personale, mezzi finanziari e tempo, quest'ultimo aspetto spiega perché si è dovuto rinunciare a tenere una seduta della Camera medica nel dicembre 1999, come previsto inizialmente. Sono state condotte delle discussioni intense tra i partner su questioni essenziali per l'avvenire delle tariffe come il concetto del valore intrinseco delle prestazioni («Dignität»), della futura gestione delle tariffe ("handling") e delle future convenzioni che si baseranno sulla struttura tariffaria e intorno ad essa (tariffa AINF/AM/AI; convenzioni tra il Concordato e la FMH sulle condizioni di inquadramento dell'introduzione della nuova struttura tariffaria nei cantoni). La neutralità dei costi ha occupato naturalmente anche i partner, mentre la questione centrale su questo oggetto era quella del valore del punto al momento dell'entrata in vigore della struttura tariffaria. Altro punto importante dibattuto: l'organizzazione che succederà alla Commissione TarMed (che, una volta conclusi i suoi lavori, non ha più ragione d'essere).

Abbordando in seguito la questione sugli studi sul terreno ("Feldstudien») e sulle prove pilota ("Pilotstudien»), il presidente desidera dare delle precisazioni che gli sembrano indispensabili. La nozione di studio sul terreno è relativamente aperta e si applica a delle situazioni in cui chi fornisce cure fattura secondo la nuova tariffa, ma la tariffa vecchia rimane l'unica applicabile. Questo genere di studi è già stato condotto e lo è ancora adesso, ed è in grado di fornire alcune informazioni utili. Le prove-pilota, invece, consistono nell'applicazione della nuova tariffa in un ambito temporale ben definito; questo genere di prove ha senso solamente se tutti gli attori (medici di città, medici ospedalieri, cantoni) vi partecipano. Inizialmente era stato previsto di effettuarne in alcuni cantoni (8), ma si è dovuto rinunciarvi in mancanza della volontà di tutte le entità interessate e/o in mancanza di mezzi finanziari. La TarMed ha dovuto costatare che questo genere di prove-pilota non era praticabile e non ha quindi nessun senso volerne esigere.

Il presidente prosegue la sua introduzione alle deliberazioni chiedendo la comprensione da parte dei delegati per il fatto che il Comitato Centrale si attende da loro che indichino quali debbano essere le posizioni da difendere per la FMH sui punti vicini alla struttura tariffaria come la neutralità dei costi, i contratti ancora da concludere e la posizione da adottare, se necessario, nei confronti di alcune istanze statali, senza disporre di testi già maturi per una decisione definitiva. 
Il presidente dichiara in seguito che è stata effettuata una valutazione interna sul risultato di queste ultime trattative tra partner: questa valutazione ha avuto un grande ruolo per la redazione finale della versione "alfa 2.2». Una valutazione esterna è pure prevista e le sue conclusioni saranno consegnate in breve e potranno essere utilizzate già per il round di revisione 2000 per quel che concerne i fattori che costituiscono parte integrante della prestazione tecnica, in particolare nel settore ospedaliero. L'incarico dovrebbe essere affidato ad un istituto estero specializzato nelle questioni economiche, tariffarie e di gestione ospedaliera.

Il presidente conclude questa sua introduzione facendo capire in tutta chiarezza che la decisione attesa oggi dalla Camera medica non è una votazione sulla neutralità dei costi, ma una votazione sulla nuova struttura tariffaria. Questa votazione dovrà essere chiara, poiché l'alternativa è "sì oppure "no", perché un "sì, ma" sarà considerato un "no". Ricorda che i termini legali sono scaduti da tempo e che tutti i contratti (tariffa AINF/AM/AI, convenzioni cantonali, Catalogo delle prestazioni ospedaliere) sono stati disdetti. Detto ciò, viene ripetuto ancora una volta che la votazione sulla struttura tariffaria non impedisce in nessun modo che siano dati al Comitato Centrale e alla sua delegazione alle negoziazioni delle indicazioni per quel che concerne le posizioni da difendere sulle importanti questioni adiacenti ancora aperte (neutralità dei costi, convenzione-quadro, ecc.).

\section{Concezioni e convenzioni complementari sulla struttura tariffaria versione "alfa 2.2 "}

Prima di passare alla discussione sul concetto di neutralità dei costi, la Camera deve occuparsi di una proposta del dott. $R$. Streit, presidente della Società dei medici del canton Berna, ( ${ }^{\circ} 1$ ai punti 3.5 e 6 ) il cui $\mathrm{III}^{\circ}$ paragrafo, se accettato, equivarrebbe ad un rinvio generale dei concetti e delle convenzioni proposte al voto dei delegati alle cifre 3.1 e 3.7 di questo punto 3 dell'ordine del giorno e avrebbe come conseguenza logica che si passa immediatamente a discutere il punto seguente.

Il dott. $R$. Streit dichiara di rappresentare il parere di numerosi membri parlando a favore del rigetto di testi sui quali non si è ancora finito di negoziare. Quest'anno più di mille posizioni attendono una revisione, si stanno rimaneggiando due capitoli, le remunerazioni relative alle prestazioni chirurgiche e intervenzionali devono essere ancora riviste. Come fare a legarsi in un tale fascio di incertezze, si chiede l'intervenuto, che considera del tutto irresponsabile pensare di passare ad una votazione generale sulla struttura tariffaria mentre ci troviamo sotto la spada di Damocle della neutralità dei costi e quando la questione della remunerazione per la distribuzione dei medicinali indipendentemente dal margine commerciale (MUMA) non ha ancora trovato soluzione. Il dott. R. Streit teme che un'accettazione della strut- tura tariffaria alfa 2.2 sotto questi auspici apra direttamente la via, tramite un budget globale il cui pericolo non è per niente scartato, al razionamento delle cure di cui i pazienti saranno i primi a patire. Fa appello al senso di responsabilità dei delegati e li invita a non lasciarsi mettere sotto pressione dal fattore tempo.

Il presidente risponde che la versione "alfa 2.2" non è così aperta come l'intervenuto fa credere. La maggior parte è fissata e rimane solo qualche centinaio di prestazioni che devono ancora essere corrette. Prima dell'entrata in vigore della struttura tariffaria, sarà possibile rivedere la nomenclatura per la ginecologia e procedere ad una revisione economica degli istituti di radiologia. Per quel che concerne le proposte della FMS, saranno esaminate alla luce dei dati della struttura tariffaria TarMed, e ciò significa che una volta d'accordo sui principi, i calcoli possono essere fatti in qualche minuto. Vediamo ora le conseguenze derivanti dall'accettazione della proposta del dott. Streit: non ci sarà più la possibilità di fare un giro di revisione; non ci sarà più il modello FMS e probabilmente non ci sarà più nient'altro, se non l'attesa delle decisioni delle istanze ufficiali. D'altra parte il Comitato Centrale si è sforzato di integrare nella sua proposta le proposte supplementari che ci sono pervenute, come la lettura della suddetta proposta permette a ciascuno di rendersene conto. Inoltre, non ci troviamo in una guerra di posizione, ma in una guerra di movimento e quindi in un processo evolutivo nel quale sono già stati ottenuti dei miglioramenti sostanziali.

Nella discussione generale che segue, il dott. $W$. Grete, presidente della Società zurighese di medicina, fa capire che ci si è riuniti oggi non per approvare una tariffa definitiva, ma per manifestare la nostra intenzione a continuare a negoziare. Ciò potrà essere fatto, tuttavia, solamente se oggi diciamo molto chiaramente "si" alla struttura tariffaria. È importante che diamo questo messaggio di fiducia da parte del corpo medico. Il dott. Th. Schweizer, BE, da parte sua, riconosce che la tariffa GRAT è una buona cosa, ma nella questione della neutralità dei costi vede un vero e proprio virus che lasceremo penetrare nel sistema e che, tramite i politici, condurrà direttamente al razionamento delle cure, cosa che respingiamo.

$\mathrm{Si}$ passa allora alla votazione sul $I I I^{\circ}$ capoverso della proposta del dott. $R$. Streit («I concetti e le convenzioni presentati oggi, notevolmente immaturi, soprattutto il concetto della neutralità dei costi, sono respinti»). Questa proposta è respinta a chiara maggioranza.

\subsection{Posizione della FMH sulla neutralità}

dei costi / Concetto d'introduzione

(compresa l'applicazione della proposta Ramstein) Questa questione è ancora l'oggetto di intense discussioni e ai delegati è stato consegnato un documento che presenta la situazione del fascicolo al 30 gennaio 2000. Il presidente fa osservare che sarebbe errato considerare questa questione in modo unilaterale, poiché questa nozione offre anche una prote- 
zione verso il basso e che, da questo punto di vista, gli assicuratori sono pure vincolati legalmente, in particolare nel settore della LAMal. È stato fermamente affermato il rifiuto da parte della FMH di vedere la neutralità dei costi utilizzata per introdurre un budget globale in modo indiretto. Non si può più parlare di budget globale, visto che il valore centrale considerato sarà quello del costo per assicurato. A ciò si aggiunge che ci si sta dirigendo verso la definizione di un tempo di osservazione "ragionevole». Gli assicuratori comprendono con noi che si tratta di introdurre le nuove tariffe in modo tale che non conducano ad un aumento dei costi pro capite né, nell'interesse dei pazienti, ad un razionamento delle cure.

Il dott. U. Nägeli, presidente della Società di medicina del canton Glarona, espone i motivi della sua proposta (n. 2 ad tractandum 3.1) che chiede che «nella misura del possibile, la FMH deve sforzarsi di ottenere che il valore del punto tariffario nel settore della LAMal sia uniforme quando viene introdotto 0 , più precisamente, che si situi nell'ordine di $+/-4 \%$, deciso nel corso della seduta della Camera medica dell'8 aprile 1999». Dal momento in cui è stata depositata la sua proposta, il suo autore costata che ci si è allontanati sempre di più da questa decisione dell'aprile scorso, sotto le pressioni esercitate da parte politica. Esigendo la neutralità dei costi, viene pervertita l'idea stessa di una tariffa costruita sull'analisi d'economia dell'azienda. Il dott. U. Nägeli, che dice di temere una perdita di solidarietà in seno al corpo medico, si basa da parte sua sul principio che lo stesso lavoro merita lo stesso stipendio. Ci si può chiedere se si ha già perso la fiducia nei meccanismi di correzione introdotti nella TarMed per eliminare delle differenze troppo grandi. D’altra parte permangono grandi incertezze nei settori annessi (MUMA, elenco delle analisi, ecc.).

Il dott. H. H. Brunner fa osservare che è la tecnica delle negoziazioni che ha fatto si che alcuni temi siano stati messi tra parentesi. Poiché si tratta della MUMA, la FMH e il Concordato sono d'accordo per prendere la MUMA come posizione tariffaria all'interno della struttura tariffaria. Il problema non sta, tuttavia, - una volta di più - presso i partner contrattuali, ma a livello delle autorità federali, dove si sta ancora discutendo per sapere se la questione della distribuzione dei medicinali deve essere regolata a livello della legge o nell'ambito di una revisione d'ordinanza d'applicazione della LAMal. Le decisioni in proposito non sono ancora state prese e tutto lascia pensare che non saranno prese quest'anno. Il presidente chiede che nel verbale venga scritto chiaramente che il Comitato Centrale considera impensabile, per considerazioni relative alla neutralità dei costi, che si possa pensare all'introduzione delle nuove tariffe senza che sia regolata la questione della MUMA.

Parla a favore della proposta del dott. Nägeli anche la sua consorella, la dott.ssa C. Hefti, GL, che evoca le difficoltà incontrate già oggi per trovare dei medici svizzeri che desiderino riprendere degli studi medici favorevolmente situati nel suo cantone. La
TarMed non dovrebbe aggravare ancora di più la mancanza di attrattiva delle regioni periferiche.

Il presidente costata che la proposta (n. 5 ad tractandum 3.1), presentata dal dott. F. Hasler, presidente della Società di medicina del cantone dei Grigioni, è molto vicina alla proposta del dott. Nägeli e suggerisce che sia trattata simultaneamente. Il dott. Hasler non vi si oppone, facendo notare che la sua proposta, come quella del dott. Nägeli e quella del Comitato Centrale, che chiedono una convergenza a termine (n.1 al punto n. 5), vanno nella stessa direzione. Un altro motivo che parla a favore di un restringimento della forcella del valore del punto ovvero per una tariffa federale è presentata dal dott. M. Röthlisberger, $G R$, che evoca la frequenza con la quale i medici di un cantone devono occuparsi di pazienti di altri cantoni.

Tocca quindi al dott. Bl. Bourrit, presidente dell'Associazione dei medici del Canton Ginevra, motivare la sua proposta (n. 3 ad tractandum 3.1) che chiede in sostanza che la Camera medica debba rifiutare la TarMed, se il concetto di neutralità dei costi dovesse essere mantenuto in modo da costituire un budget globale. Il dott. Bourrit capisce sino ad un certo punto che gli assicuratori cerchino di evitare che l'introduzione della tariffa provochi un aumento dei costi, ma è la neutralità a lungo termine 0 , in altre parole, il mantenimento della durata di costrizioni, che costituisce un problema. Se i ginevrini sono pronti ad accettare la TarMed come struttura tariffaria, rifiutano una neutralità dei costi, anche su cinque anni.

Il presidente H. H. Brunner deve fornire qui diverse precisazioni e considerazioni sulle sottigliezze di una negoziazione complessa, sulle quali sembra necessario mantenere una certa discrezione nell'ambito della presente recensione. Notiamo semplicemente che il concetto di neutralità dei costi nella versione 5.0, che era stata rifiutata dalla Camera medica nel giugno 1999, non è più d'attualità e che è su un altro testo che si continuano attualmente le discussioni, non in seno alla TarMed, ma su un piano bilaterale, direttamente con gli assicuratori e sulla base della versione 4.0.

L'ultima proposta (n. 4 ad tractandum n. 3.1) sulla questione della neutralità dei costi viene dal dott. E. Steinmann, presidente della Società di medicina del canton Lucerna, che la presenta utilizzando dei fogli sul proiettore. Nella sua proposta in 5 punti, l'autore chiede che "in un lasso di tempo di 5 anni i valori del punto tariffario delle tariffe TarMed dovranno essere unificati sino al punto in cui le differenze regionali e cantonali ancora esistenti si baseranno solamente su delle particolarità economiche regionali e cantonali riconosciute" (punto 1). L'autore prevede al punto 2 della sua proposta che "a partire dall'introduzione delle strutture tariffarie, il valore del punto delle prestazioni tecniche debba essere fissato uniformemente per l'insieme della Svizzera ed essere in seguito adattato nella misura in cui la situazione economica l'esiga dal punto di vista della gestione aziendale. Nell'ambito della neutralità dei costi sono escluse 
delle modifiche». Gli ultimi tre punti della proposta preconizzano una clausola particolare per i medici che possono fatturare solamente la prestazione medica, lo studio della fattibilità di una "rete» per 14-16 cantoni che hanno una struttura di costi simili e infine, che la FMH sia incaricata di assicurarsi che il principio di un valore uniforme del punto sia applicato a tutti i settori di attività della professione. Il dott. Steinmann è guidato sia dalla preoccupazione che le disparità troppo importanti del valore del punto ai livelli regionale e cantonale silurino l'idea iniziale del GRAT come anche da quella di dare un tempo ragionevole d'adattamento sino a quando la convergenza preconizzata possa essere realizzata.

Il presidente $H$. H. Brunner fa osservare che non sarebbe per niente realista voler realizzare la convergenza all'inizio dell'introduzione della tariffa, salvo voler suscitare delle tensioni importanti in seno al corpo medico e alla FMH e creare dei problemi insormontabili a numerose casse malattia. Le opposizioni politiche ad una tale convergenza sono pure note a tutti, almeno a breve termine. Una concretizzazione di quest'idea a più lunga scadenza è invece pensabile. Si dovrebbe, in questo caso, procedere ad una ridistribuzione a tappe e scegliere se si vuole operare sulle componenti della prestazione tecnica o della prestazione medica. Sono questi i punti da abbordare nella discussione, conclude il presidente.

Viene aperta la discussione generale dapprima dal dott. W. Grete, $Z H$, che fa notare che le soluzioni liberali, più flessibili, sono da preferire a delle soluzioni centralizzatrici. Non è né a Berna né a Soletta che troviamo i nostri alleati, ma in ognuno dei nostri cantoni, incominciando dai cantoni stessi, nella loro qualità di responsabili del buon funzionamento dei loro ospedali. Il presidente della Società vodese di medicina, il dott. D. Laufer, appoggia il dott. Grete, facendo osservare che i medici sono i soli a sapere quel che guadagnano (e guadagneranno), mentre solamente degli studi dettagliati sul terreno saranno in grado di definire l'evoluzione dei costi in futuro. I costi non sono determinati unicamente dai medici, ma anche dagli ospedali, dai policlinici e dal comportamento dei pazienti. Le cose devono regolarsi, per lo meno all'inizio, nei cantoni. Detto ciò, il dott. D. Laufer appoggia inoltre l'iniziativa del dott. E. Steinmann, affinché il Concordato possa chiedere delle spiegazioni razionali sulle differenze di costo persistenti tra un cantone e l'altro; un periodo di 5 anni sembra ragionevole per poter procedere ad eventuali adattamenti, come anche per trovare una soluzione al grave problema della profarmacia.

Il dott. J. Ammann, della Società svizzera di chirurgia e presidente della FMS, pensa che la discussione stia diventando oziosa, nella misura in cui si dispone ora di un parere di diritto, le cui conclusioni sono state convalidate da eminenti giuristi. Solo i cantoni, hanno de lege lata, la competenza per approvare le convenzioni sul valore del punto LAMal sul loro territorio. Il dott. H. H. Brunner non ignora queste considerazioni, ma fa notare che non si può liquidare il problema così facilmente, in particolare perché il Consiglio federale ha la competenza di pronunciarsi sul fondo in caso di procedura di ricorso in un cantone. Il dott. E. Steinmann, LU, si appella ancora una volta alla solidarietà, mentre il dott. $R$. Streit, BE, vede nelle tesi che si affrontano qui un argomento supplementare per nutrire i suoi timori espressi in precedenza. Teme, in particolare, che l'indispensabile clausola del "ceteris paribus" cada se la fase d'introduzione neutrale dovesse essere riportata ad un anno come viene postulato dal Comitato Centrale alla cifra 3 della sua proposta $n$. 1 al punto 3.1. Non dobbiamo quindi accettare niente senza la possibilità di apportare delle correzioni. Secondo il dott. A. Haefeli, presidente della Società di medicina del Canton d'Argovia, ci troviamo di fronte al dilemma neutralità dei costi oppure budget globale. Questo dilemma può essere risolto solamente se la fase d'osservazione della neutralità dei costi è il più breve possibile e gli strumenti per misurarla sono ragionevoli; questo deve essere l'oggetto delle nostre negoziazioni con gli assicuratori. Dopo aver evocato qualche altro argomento, che gli sembra di buon augurio per questa negoziazione, il dott. Haefeli viene alla proposta del dott. Steinmann che postula la convergenza dei valori dei punti. In quanto presidente del gruppo di lavoro delle società cantonali di medicina, il dott. A. Haefeli è del parere che la Camera medica non dovrebbe imporre alle organizzazioni cantonali delle ingiunzioni che esse non vogliono e non possono seguire. Un termine di 5 anni sembrerebbe ragionevole sulla carta per realizzare la convergenza, ma ciò significherebbe per numerosi confratelli e consorelle, in numerosi cantoni "cari" che non sono tutti forzatamente posti sulle rive del Lemano, un rivoluzionamento delle strutture e delle abitudini tali da causare una rivoluzione vera e propria. Il dott. $R$. Nyffeler, presidente della Società di medicina del Canton Friburgo, fa notare che la struttura tariffaria unica iscritta nella legge ha soprattutto come ragione d'essere quella di poter controllare i costi. Dobbiamo quindi essere ben coscienti del fatto che se non abbiamo la possibilità d'analizzare noi stessi le nostre strutture di costi, ci sarà impossibile negoziare i nostri valori di punto e giustificare i costi che causeremo.

Poiché la discussione generale è terminata con quest'ultimo intervento, il presidente $H$. H. Brunner procede ora alla liquidazione di diverse proposte relative alla proposta n. 1 del Comitato Centrale al punto n. 3.1, prima di poter passare alla votazione sulla suddetta proposta. Fa osservare ai delegati che il Comitato Centrale si è sforzato di includere nella sua propria proposta numerose proposte presentate. $\mathrm{Fa}$ in particolare notare la cifra 2 della proposta del Comitato Centrale ("La Camera medica dichiara che la struttura tariffaria TarMed - attualmente versione alfa 2.2 - avrà effetto sulle tariffe solamente quando un accordo contrattuale concernente la neutralità dei costi all'introduzione delle tariffe sarà stata trovata tra gli assicuratori competenti, da una parte, e la FMH, dall'altra."), facendo osservare che la Camera medica non potrebbe esigere oggi di più. Il secondo 
punto sul quale la Camera medica dovrà pronunciarsi è quello di sapere se vuole o non vuole la convergenza secondo la proposta del dott. Steinmann. Il presidente attira l'attenzione dei delegati sul fatto che con un termine di 5 anni, si potrebbe dover registrare l'entrata in vigore degli accordi bilaterali in seguito ai quali, con la tendenza uniformatrice ben nota, le decisioni che prendiamo oggi non avrebbero valore. Si dovrebbe quindi chiedere se non sarebbe meglio studiare la possibilità di una convergenza, cosa che si prevede anche al punto 3 della proposta del Comitato Centrale. Punto importante da aggiungere alla proposta del Comitato Centrale: quello della remunerazione per le prestazioni relative alla distribuzione dei medicinali (MUMA). A queste condizioni, e sempre che si unisca, come lo richiede il dott. R. Nyffeler, l'elenco delle analisi per i cantoni che non l'hanno ancora, il dott. U. R. Nägeli, GL, si dice pronto a fondere la sua proposta n. 2 ad tractandum n. 3.1 nella proposta del Comitato Centrale, perché si avrebbe cosi la garanzia che il Comitato Centrale si sforzerà di far passare il principio della convergenza in un certo lasso di tempo. Il dott. H. H. Brunner assicura che quando la questione sarà stata studiata, verrà messa sul tavolo delle negoziazioni con il Concordato. Il dott. F. Hasler, GR, si affianca da parte sua all'idea espressa dal dott. U. Nägeli per quel che concerne la sua proposta $\mathrm{n} .5$ ad tractandum n. 3.1.

Si passa quindi alla votazione sulla proposta n. 3 ad tractandum n. 3.1 presentata dal dott. Bl. Bourrit, $G E$, e della quale si è parlato qui sopra. La suddetta proposta viene respinta a grande e chiara maggioranza.

Viene quindi votata la proposta n. 4 ad tractandum n. 3.1, presentata dal dott. E. Steinmann, $L U$. Anche questa viene respinta (91 voti contro 54). Il presidente commenta questa votazione dicendo che secondo lui aveva un valore indicativo, ma che la questione della convergenza resta ancora sul tavolo, conformemente a quanto è stato detto in precedenza.

Si passa in seguito alla votazione sulla proposta n. 1 ad tractandum n. 3.1 del Comitato Centrale sulla neutralità dei costi, alla quale sono stati aggiunti gli elementi apportati nel corso della discussione per quel che concerne la MUMA e l'elenco delle analisi di laboratorio. Questa proposta è accettata con 151 voti favorevoli e 9 contrari.

\subsection{Applicazione del valore intrinseco qualitativo e} 3.3 Revisione 2000

Viene data la parola al dott. M. G. Schwöbel, della Società svizzera di chirurgia pediatrica, per esporre la sua proposta n. 2 ad tractandum n. 3.2 che chiede che "la menzione (chirurgia pediatrica debba figurare sotto le prestazioni particolari effettuate dai chirurghi pediatrici». L'autore di questa proposta è cosciente del fatto che possa apparire ad alcuni come una questione di dettaglio, ma riveste un valore fondamentale per la sua società specialistica. Fa valere che il valore intrinseco qualitativo "chirurgia pediatrica" figura solamente 4 volte tra le 4000 posizioni, mentre tutte le altre posizioni sono "annegate» in una posizione comune che non è sufficiente; in effetti numerosi interventi rientrano esclusivamente nel campo della chirurgia pediatrica e queste prestazioni sono perse se vengono menzionate solamente in modo implicito in un preambolo. Di conseguenza, i chirurghi pediatrici chiedono che sia lasciata loro la possibilità di verificare tutte le posizioni se praticano questo genere di interventi e di aggiungervi l'indicazione "chirurgia pediatrica». Il dott. H. H. Brunner, anticipando sulla proposta seguente n. 3 ad tractandum 3.2. presentata dalla stessa società specialistica (che chiede un rimaneggiamento redazionale della cifra 77 delle interpretazioni generali), considera che i casi sollevati dal dott. Schwöbel nelle sue due proposte hanno a che fare prima di tutto con la tecnica della nomenclatura; in effetti si è cercato di semplificare le cose, perché c'erano poche società specialistiche, a parte quelle dei chirurghi pediatrici, che avevano altrettante prestazioni in tutti i capitoli e che non sarebbe stato pertinente introdurre dei limiti. Sarebbe errato vedervi un qualsiasi discredito dell'attività dei chirurghi pediatrici. Non ci sarà quindi opposizione né da parte del Comitato Centrale né da quella della TarMed per accettare queste due proposte nell'ambito del round di revisione 2000. Malgrado queste spiegazioni, il dott. Schwöbel vuole che la Camera medica voti sulle due proposte, allo scopo di stabilire in modo del tutto chiaro che i chirurghi pediatrici abbiano gli stessi diritti delle altre società specialistiche della FMH.

Si passa allora alla votazione, separatamente, sulle proposte n. 2 e 3 ad tractandum n. 3.2. Queste due proposte sono accettate, una dopo l'altra, alla quasi unanimità.

Il dott. M. Battaglia, copresidente dell'ASMAC, considera che si può si prendere conoscenza dei concetti presentati, ma che sarebbe prematuro accettare sin d'ora, quando non è stata ancora data l'occasione di discutere sui risultati concreti, in particolare sulla questione dei diritti acquisiti e sullo statuto di chi ha il titolo FMH rispetto a chi non l'ha. L'applicazione del concetto di valore intrinseco è altrettanto importante per i giovani medici che quello della neutralità dei costi per i medici che esercitano da tempo. L'ASMAC può dare il suo accordo alla struttura tariffaria, accetta il sistema del "work in progress", ma non potrebbe dare il suo avvallo ad un concetto che è all'inizio della sua evoluzione, cosa che il presidente contesta, concedendo tuttavia che si debbano ancora prendere delle misure, segnatamente a titolo transitorio.

Il dott. L. Dubs, presidente della Società svizzera d'ortopedia, costata che le due proposte che aveva presentato entro i termini a proposito del tractandum 3.2 non hanno trovato posto nel testo della proposta del Comitato Centrale; annuncia che riprenderà queste proposte nell'ambito della discussione concernente il round di revisione 2000 , visto che sono in gioco, in particolare, degli errori di sistematica nell'applicazione del valore intrinseco («Dignität»).

Dopo che il presidente ha tenuto a precisare che tutti gli errori, senz'altro correggibili, non derivano 
necessariamente da macchinazioni o volontà arbitraria, si passa alla votazione sulla proposta del Comitato Centrale n. 1 ad tractandum 3.2. Questa proposta in tre punti domanda in sostanza alla Camera di prendere conoscenza del concetto di «Applicazione del valore intrinseco qualitativo" del 23 dicembre 1999 e di approvarlo con riserva di concretizzazione delle proposte trasmesse in occasione della Conferenza dei presidenti del 13 gennaio 2000. Inoltre, il Comitato Centrale dovrà informare regolarmente la Camera sull'applicazione di questo concetto e assicurarsi di proteggere in modo rigoroso i dati, in particolare affinché nessuna banca dati elettronica contenente delle informazioni relative ai membri non vada in mano a terzi. Questa proposta è accettata a forte maggioranza.

La proposta n. 1 al tractandum n. 3.3, presentata dal dott. R. A. Steiner, presidente della Società svizzera di ginecologia e ostetricia, che chiede che si definisca, con le società specialistiche chirurgiche, un settore "sala d'operazione dello studio medico" che non sia estranea alla pratica privata, è accettata a maggioranza chiara, tanto più che il presidente $H$. H. Brunner l'aveva accettata a nome del Comitato Centrale.

Si passa in seguito alla proposta n. 2 ad tractandum 3.3 deposta dal prof. T. Rüedi, presidente della Società svizzera di chirurgia, e presentata nel corso della seduta dal dott. J. Ammann. Questa proposta, sostenuta dalla FMS, chiede in sostanza che si fissi contrattualmente tra tutti i partner che i risultati del round di revisione 2000 siano aggiunti alla struttura tariffaria TarMed. Il dott. Ammann vede nell'accettazione di questa proposta un modo per placare i timori espressi all'inizio della seduta dal dott. R. Streit. Il dott. H. H. Brunner, a nome del Comitato Centrale, accetta questa proposta. Segnala che il round di revisione 2000 fa parte della struttura tariffaria, che questo sarà previsto nel contratto quadro con il Concordato e che infine il calendario delle negoziazioni sarà fissato, dopo questa seduta, con i nostri partner.

\subsection{Struttura dell'organizzazione che succederà alla Commissione TarMed}

Il dott. P. Neidhart, della Società svizzera d'anestesia e di rianimazione, presenta la proposta $\mathrm{n}$. 2 ad tractandum 3.4 del suo confratello, il dott. Meister. Questa proposta tende a un rigetto della "convenzione TarMed (compresi lo statuto e il regolamento dell'organizzazione dell'Associazione TarMed)». Si afferma che la forma giuridica dell'associazione non si presti ad un'organizzazione del tipo della "TarMed», perché il membro di un'associazione può sempre lasciarla, che i rapporti di voti rischiano di operarsi a detrimento della FMH e che sembra si voglia allegramente mescolare diritto contrattuale e diritto delle associazioni. Il presidente dichiara senza ambagi che dovrà opporsi a questa proposta, perché la formula che è stata ritenuta, dopo mesi di discussione, è la meno cattiva, dopo che la forma di una società semplice ha dovuto essere definitivamente abbandonata. D'altra parte le decisioni devono essere prese all'unanimità, il pericolo che sia messa in minoranza è del tutto relativo.

Dopo queste spiegazioni, la proposta $n .2$ ad tractandum 3.4 è messa in votazione ed è respinta a chiara maggioranza.

Si passa allora alla proposta n. 3 ai tractandum 3.4, 3.5, 6 e 7 presentata dal dott. J. P. Jenny, della Società svizzera d'oftalmologia, che tende a modificare lo statuto della nuova organizzazione TarMed e il testo delle convenzioni TarMed allo scopo, da una parte, (a) che un membro di ogni disciplina medica interessata possa sedere al comitato al fianco del delegato della FMH e che, d'altra parte, (b) il membro della società di specialità medica interessata da una questione tariffaria possa beneficiare dello stesso diritto di voto e delle stesse competenze del membro della FMH. Il dott. Jenny vi vedrebbe una garanzia di plausibilità contro certe aberrazioni come la sua disciplina ha dovuto registrare nel corso delle negoziazioni.

Il dott. H. H. Brunner pensa che la parte (a) della proposta non è neanche immaginabile; non si oppone, invece, alla parte (b) che concerne le delegazioni. I delegati sono concordi con questo modo di vedere, poiché rifiutano all'unanimità la parte (a) mentre accettano a forte maggioranza la parte (b).

Si passa allora alla proposta del Comitato Centrale n. 1 ad tractandum 3.4, che preferiamo citare per intero, per meglio comprendere quanto seguirà.

"1. La Camera medica prende conoscenza della situazione delle negoziazioni concernenti la nuova organizzazione TarMed e dà la sua approvazione.

2. Il CC è incaricato di applicare nelle negoziazioni in corso le decisioni prese dalla Camera medica concernenti la nuova organizzazione TarMed. Deve in particolare assicurarsi che sia adottata per principio la decisione all'unanimità.

3. I mezzi necessari al finanziamento della nuova organizzazione TarMed devono essere definiti interamente e in modo esauriente dalla Camera medica nell'ambito dei dibattiti ordinari sul budget per l'anno seguente.

4. Con riserva che $i$ punti 2 e 3 siano eseguiti, il CC è investito del potere di concludere le convenzioni necessarie."

Il dott. $R$. Streit, BE, propone di sopprimere la cifra 4 qui sopra, considerando che spetta al legislativo e non all'esecutivo concludere le convenzioni. Il presidente H. H. Brunner si oppone a questa proposta, facendo valere che queste convenzioni sono praticamente chiuse e che si attendono solamente gli allegati; non si deve, per un eccesso di sfiducia, frenare inutilmente la costituzione della nuova organizzazione. D'altra parte e a proposito della cifra 3 qui sopra, il presidente precisa al dott. F. Bossard, presidente della Società svizzera di radiologia medica, che il budget per il primo anno dovrebbe ammontare a 150000-200000 franchi al massimo.

Dopo qualche scambio verbale sulla fondatezza di una decisione immediata su questo budget, si passa alla votazione sulla proposta del Comitato Centrale, 
procedendo in due tempi. In una prima votazione, i delegati respingono la proposta del dott. $R$. Streit (soppressione della cifra 4) a chiara maggioranza. In una seconda votazione, i delegati accettano a chiara maggioranza la proposta integrale del Comitato Centrale.

\subsection{Struttura della delegazione della FMH} alle negoziazioni e modo di procedere La proposta in 4 punti presentata dal Comitato Centrale (n. 1 ad tractandum 3.5) tende a far avvallare dalla Camera il concetto "Struttura del servizio tariffario/delegazione alle negoziazioni» (1), prevede (2) che "la delegazione alle negoziazioni incaricata delle nuove tariffe delle prestazioni chirurgiche e intervenzionali secondo il modello di costi FMS del 6.12.1999 sarà composta nel modo seguente:

- direzione al delegato del Comitato Centrale

- 7 membri nominati dalla FMS

- 2 membri nominati dal Collegio dei medici di famiglia (CMPR)

- 1 membro nominato dalla Foederatio Medicorum Curantium (FMC)».

La proposta chiede inoltre (3) che «la revisione 2000 sia trasmessa alla delegazione alle negoziazioni della nuova organizzazione TarMed" e che "prendano parte a tutte le negoziazioni, con tutti i diritti e doveri, al massimo tre delegati della società di medicina competente/che presenta la proposta, a carico di quest'ultima" e prevede (4) che i 10 membri del consiglio consultivo delle società specialistiche saranno eletti alla Camera medica del giugno 2000.

Il dott. B. Meister, presidente della Società svizzera di anestesia e rianimazione, si vede offrire l'occasione per presentare, a parole e con grafici, le cause profonde che hanno condotto all'elaborazione del modello di costi FMS. Illustra ricorrendo ad esempi eloquenti alcune insufficienze della struttura TarMed e presenta brevemente quelli che potrebbero essere i mezzi per rimediarvi e impedire che i medici accreditati non finiscano semplicemente per scomparire, cosa di cui anche i pazienti non tarderanno a patire. La preoccupazione di evitare una frattura in seno al corpo medico postula che questa proposta sia accettata, conclude il dott. B. Meister.

Il presidente si riferisce a delle comunicazioni anteriori dalle quali, già allora, risultava che la remunerazione e la ponderazione di una parte delle prestazioni chirurgiche e intervenzionali non erano per niente ottimali, in particolare per numerosi interventi effettuati con un coefficiente di valore intrinseco inferiore. Questo problema è stato progressivamente riconosciuto dai negoziatori e, anche presso gli assicuratori esiste la volontà di rimediarvi. Si deve sottolineare che il modello FMS offre una base valida per procedere a queste correzioni, in particolare perché opera con degli elementi della struttura TarMed, cosa che, tecnicamente, rappresenta un grande vantaggio. D'altra parte, siccome i medici di famiglia chiedono che se la Camera decidesse per la variabile del coefficiente di valore intrinseco "1.2", questa so- luzione sia limitata nel tempo e sia sostituita a termine da un altro modello, quest'ultimo modello potrà (e anche dovrà) essere ripreso dalla nuova ponderazione che risulterà dalle negoziazioni sul Modello FMS. Infine si noterà che c'è una volontà evidente di compensare uno squilibrio - identificato e definito che si è introdotto, a detrimento della ponderazione delle prestazioni invasive ed operatorie, in seno alla struttura tariffaria. Il dott. H. H. Brunner stima di dover sottolineare qui che non si deve confondere le nozioni di "prezzo della prestazione», da una parte, e di «diminuzione di reddito», dall'altra. La proposta del Comitato Centrale, se venisse accettata, costituirebbe parte integrante della proposta finale sulla struttura tariffaria alfa 2.2; è normale dunque che la FMS sia ben rappresentata nelle negoziazioni previste che dovrebbero aprirsi ben presto.

Su una domanda del dott. R. Nyffeler, FR, che desidera che si passi al più presto agli «studi del paniere tipo" piuttosto che commentare delle prospettive di perdite di reddito qui o là, il dott. H. H. Brunner prende un'ultima volta la parola su questo capitolo. Precisa che nella sua qualità di "ingegnere» che lavora a questo progetto, ha da tempo segnalato due problemi che non hanno potuto essere risolti prima che le cifre siano sul tavolo, in mancanza dell'accordo di chi risponde dei costi: quello che risiede nel calcolo della remunerazione delle prestazioni tecniche in ambiente ospedaliero (vero "ceterum censeo" da tre anni!) e quello che interessa lo squilibrio nella struttura tariffaria che fa che ci sia comprensione per la posizione della FMS. Detto ciò, mettendosi sempre nel suo ruolo d' 'ingegnere» afferma che l'accettazione di questa proposta non sia di natura tale da causare degli inconvenienti per i medici non intervenzionisti.

Si passa quindi alla votazione sulla proposta del Comitato Centrale n. 1 ad tractandum 3.5 dalla quale risulta una grande maggioranza accettante.

NB: Si passa allora alla discussione sulle proposte che figurano al punto 3.9. Si ricorderà qui che la proposta del dott. R. Streit, BE, concernente i punti 3.5 e 3.6 è già stata trattata e che delle proposte concernenti i punti 3.5, 3.6 e 3.7 depositate dal dott. J. Ammann, Società svizzera di chirurgia, dal dott. L. Dubs, Società svizzera di ortopedia, e dal dott. G.-P. Jenny, Società svizzera d'oftalmologia, saranno trattate ulteriormente nel corso della seduta, in concomitanza con il punto 5 (approvazione della versione alfa 2.2).

\subsection{Applicazione informatica}

Sono state inoltrate due proposte a proposito di questo punto all'ordine del giorno che si occupa di un argomento (trasferimento elettronico dei dati e banche dati) di cui il dott. H. H. Brunner sottolinea l'importanza capitale per l'avvenire del corpo medico.

La prima proposta (n. 1 ad tractandum 3.9) in 4 punti presentata dal dott. M. Müller-Friedli, presidente della Società svizzera di medicina generale, chiede in sostanza che (1) "ci si accordi al più presto possibile con gli assicuratori sulle modalità di fatturazione per via elettronica», che (2) la fatturazione per via elettronica non sia il pretesto per «l'introduzione 
del sistema del terzo pagante in un settore in cui il sistema del terzo garante è applicato oggi», che (3) la FMH sia incaricata di creare con gli assicuratori «dei pool di dati indipendenti ... che rispondano alle esigenze della protezione dei dati e della personalità dei pazienti e dei medici» e che si rinunci infine (4) a qualsiasi limitazione in seno alla struttura tariffaria TarMed «sino a quando tutti i medici abbiano la possibilità di fatturare elettronicamentem. L'autore di questa proposta considera della massima importanza che il corpo medico formuli lui stesso le condizioni alle quali questi trasferimenti di dati devono essere operati, tanto più che dei consulenti e informatici moltiplicano a tutto andare le loro offerte.

Il dott. H. H. Brunner dichiara che il Comitato Centrale non si oppone in nessun modo a questa proposta e questa è accettata tacitamente dalla Camera.

La seconda proposta (n. 2 ad tractandum 3.9) proviene dal dott. P. Meyrat, della Società medica del canton Soletta, e chiede che uin caso di dissenso tra responsabile dei costi e fornitore di prestazioni, quest'ultimo abbia il diritto di difendere il suo punto di vista presentando i suoi propri dati statistici".

Il dott. H. H. Brunner è in grado di rassicurare il dott. Meyrat indicando che il contratto quadro tra la FMH e il Concordato fisserà in particolare, per quel che concerne l'esame dell'economia, dell'adeguatezza e dell'efficacia dei trattamenti, dei criteri d'apprezzamento convenuti di comune accordo. La proposta del dott. Meyrat può quindi essere considerata tacitamente accettata.

NB: Si salta il punto all'ordine del giorno n. 4 (Risultato degli studi del paniere tipo/studi sul terreno) per occuparsi del punto principale di questa seduta, e cioè dell'

\section{Approvazione della versione alfa 2.2 della struttura tariffaria}

Sarà necessario un lungo percorso per giungere alla votazione finale su questo punto, poiché si deve trattare prima una serie di proposte presentate in proposito. La prima (n. 5 ad tractandum n. 5) proviene dal dott. $R$. A. Steiner, presidente della Società svizzera di ginecologia e ostetricia, che chiede, considerato le numerose incertezze esistenti ancora per quel che concerne il contenuto della struttura tariffaria, di non entrare in materia. Per il dott. R. A. Steiner le cose non sono ancora mature tanto che a tutt'oggi non è possibile dire in modo serio né "sì" né «no".

Il presidente si riferisce a quanto detto inizialmente e invita la Camera a riflettere sugli effetti di una non entrata in materia. Ciò sarà considerato come un "no" dai nostri partner in seno alla TarMed e le conseguenze che ne risulteranno sia nel settore della tariffa AINF/AM/AI sia in quello della LAMal sono state esposte senza equivoci all'inizio della seduta, in particolare il fatto che il governo, di fronte all'assenza di accordo tra le parti, dovrà fissare la struttura tariffaria. La prospettiva di un reddito di riferimento ridotto alle raccomandazioni del Sorvegliante dei prezzi, con un numero di ore sensibilmente aumentato, si avvicinerebbe a grandi passi. Non si tratta di minacce, ma di una presentazione oggettiva dello scenario più verosimile.

Non è richiesta la discussione su questa proposta, risolutamente combattuta dal Comitato Centrale, per cui si passa immediatamente alla votazione. La proposta del dott. R. A. Steiner viene respinta con 143 voti contro 19.

La Camera è quindi chiamata ad occuparsi della proposta n. 6 ad tractandum 5, depositata dal dott. M. Schwöbel, della Società svizzera di chirurgia pediatrica, che in modo molto lapidario chiede che sia respinta la versione TarMed alfa 2.2. Il dott. Schwöbel pensa, anche lui, che ci troviamo di fronte a troppe incertezze. Dice inoltre di aver perso ogni fiducia dopo aver segnalato da anni, senza effetto apparente, numerosi errori e insufficienze del progetto. In queste condizioni, se si può dire solo "sì" o "no", si sente nell'obbligo di dire "no". Il presidente H. H. Brunner non nega che i chirurghi pediatrici si siano urtati a certe difficoltà, ma fa notare che si è cercato di rimediarvi. Il dott. J. Ammann, FMS, apporta il suo sostegno ai parenti poveri della chirurgia che sono i chirurghi pediatrici e suggerisce come compromesso che li si metta tra parentesi nella struttura tariffaria come è il caso per i ginecologi e i radiologi. Il presidente Brunner si vede costretto a respingere questo suggerimento, indicando che il pacchetto di revisione 2000 è già completo, ma che i chirurghi pediatrici potranno far valere alcuni dei loro desideri nell'ambito del modello FMS.

Si apre una discussione sul modo in cui le negoziazioni sono state portate avanti e per sapere se la base delle società specialistiche è stata implicata in modo sufficiente o no in questo processo, cosa che porta il presidente a mettere qualche punto sulle «i» e la dott.ssa C. Wehren, BS, a invitare la Camera a votare infine la struttura TarMed alfa 2.2.

Prende allora la parola, il nostro invitato, il dott. M. Ganz, presidente dell'Associazione svizzera dei medici indipendenti che lavorano in cliniche private. Ringrazia il Comitato Centrale di aver sostenuto nelle colonne dell'organo professionale il sistema del medico accreditato e di aver parlato a favore del fatto che il medico accreditato con un tasso d'attività normale a carico dell'assicurazione sociale possa vedere la sua esistenza assicurata dalle nuove tariffe. Detto ciò fa notare le prospettive poco incoraggianti che risultano da uno studio attento della versione della TarMed alfa 2.2 effettuata in seno alla sua società. Tutto fa pensare che il medico accreditato, soprattutto l'operatore, sarà eliminato dal gioco delle tariffe, se non immediatamente, almeno in un periodo di 5-6 anni dopo la sua introduzione. Inoltre una parte essenziale delle prestazioni operatorie nella medicina di famiglia potrà essere praticata solamente negli ospedali sovvenzionati. Le cliniche non sovvenzionate dovranno rinunciarvi, per cui le liste d'attesa negli ospedali pubblici sono sin d'ora preprogrammate. E non si parlerà della qualità. Cosa positiva, però, e di cui si può ringraziare il Comitato Centrale: grazie alla 
garanzia dei diritti acquisiti, i medici che hanno oggi un'attività di medico accreditato avranno il tempo di adattarsi.

Dopo alcuni interventi tra i rappresentanti di alcune società specialistiche e il presidente, il dott. Schwöbel prende un'ultima volta la parola prima della votazione sulla sua proposta sottolineando che il suo intervento non è guidato da considerazioni di ordine pecuniario, ma dalla preoccupazione di vedere una società specialistica semplicemente e puramente scomparire.

É giunto allora il momento di passare alla votazione sulla proposta n. 6 ad tractandum n. 5. La Camera respinge questa proposta (135 no, $30 \mathrm{si})$.

Si continua a trattare il punto 5 dell'ordine del giorno. Il presidente costata, con l'accordo del Dott. B. Hanimann, presidente della Società svizzera di chirurgia pediatrica, che la sua proposta $\mathrm{n} .7$ ad tractandum n. 5 è diventata ormai priva di oggetto.

In una proposta $n .8$ ad tractandum n. 5, la Società svizzera di ginecologia e ostetricia, tramite il suo presidente il dott. $R$. A. Steiner, chiede che i capitoli 22 "ginecologia e ostetricia" e 23 "posizioni ginecologiche importanti» siano esclusi dalla votazione concernente la struttura tariffaria alfa 2.2. Il presidente H. H. Brunner chiede alla Camera, per motivi soprattutto sistemici, di respingere formalmente questa proposta. Si passa alla votazione che si conclude con una grande maggioranza di voti a sfavore.

Si tratta ora la proposta $\mathrm{n} .10$ ad tractandum n. 5, presentata dal presidente della Società svizzera di medicina generale, il dott. M. Müller-Friedli. Una gran parte dei postulati di questa proposta in 7 punti principali era già stata discussa alla Conferenza dei presidenti ed è stata integrata in seguito nel testo della proposta del Comitato Centrale. Il dott. Müller-Friedli ricorda che i medici di famiglia hanno potuto accettare solamente con riserva la rivalorizzazione delle prestazioni di interventi e quelle operatorie come si è fatto passando dalla versione beta 3 alla versione alfa. La proposta della SSMG equivale quindi ad un sostegno alla FMS, chiedendo nel contempo che si renda plausibile nello spazio dei prossimi due anni tutte le prestazioni rivalorizzate. Il dott. M. Müller-Friedli chiede anche che si sfruttino questi due anni per riunire dei dati che permettano infine di fare una separazione tra i redditi nel settore dell'assicurazione sociale e quelli del settore contrattuale. Il dott. MüllerFriedli chiede anche che i medici di famiglia (SSMG, SSMI, SSP) siano associati alle discussioni di esperti sul rimaneggiamento del capitolo "ginecologia e ostetricia" come anche a quelle concernenti le tecniche di diagnostica per immagini.

Il presidente annuncia che procederà alle operazioni di voto su questa voluminosa proposta solamente sui punti che sono oggetto di contestazione, mentre gli altri punti possono essere considerati accettati. Questo modo di procedere viene tacitamente accettato dai delegati. In seguito ad un intervento della dott.ssa A.-C. Froidevaux, GE, che si interroga sulla fondatezza dell'ingerenza dei medici di famiglia che chiedono di potersi sedere alla tavola delle ne- goziazioni sulle prestazioni di alcune specialità mediche, il presidente risponde che si tratta di una pratica che ha preso piede da anni in occasione delle discussioni tra esperti e che si è spesso rivelata positiva in quanto ciò ha permesso spesso di trovare il consenso. Il dott. R. A. Steiner, presidente della Società svizzera di ginecologia e ostetricia, seguito nel corso della discussione dal prof. M. Litschgi, presenta le stesse difficoltà a proposito del punto 2 della proposta n. 10 dei medici generici ad tractandum n. 5, temendo un "diktat" esterno sulle questioni che li concernono in modo specifico, questo tanto più che non hanno mai potuto beneficiare della reciprocità nelle negoziazioni delle altre società "vicine». Il dott. $B l$. Bourrit, GE, che si esprime nella sua qualità di ginecologo non può impedirsi di rivelare che tutto ciò rientra in un contesto più vasto, se si pensa alla sorte riservata l'anno scorso al programma di perfezionamento professionale dei ginecologi alla Camera medica. Ancora qualche discussione prima che il dott. $J$. de Haller, SSMG, a cui viene accordato l'ultima parola su questa controversi, dichiari che la proposta della sua società è da considerare come un tutto che la SSMG non accetta di veder distrutto.

Si passa in seguito alle operazioni di voto. In una prima consultazione, la Camera respinge con 100 voti contrari e 45 favorevoli di sopprimere il punto 2 della proposta della SSMG. Nella votazione finale, la proposta integrale della SSMG è accettata a maggioranza evidente.

Si passa in seguito alla proposta n. 11 ad tractandum n. 5, presentata dal prof. T. Rüedi, della Società svizzera di chirurgia. Questa proposta, presentata alla seduta dal dott. J. Ammann (FMS), chiede che "le tariffe di assicurazione sociale (AINF/AM/AI e LAMal) debbano obbligatoriamente includere, al più tardi al momento dell'entrata in vigore, il modello tariffario della FMS per il calcolo delle prestazioni invasive e chirurgiche». Come fa notare il presidente che si riferisce alle discussioni anteriori a proposito del modello dei costi FMS, il Comitato Centrale è cosciente del fatto che le negoziazioni a questo proposito debbano aprirsi immediatamente. La Camera accetta tacitamente la proposta del prof. T. Rüedi, come lo chiede il presidente.

In una proposta n. 9 ad tractandum n. 5, il dott. F. Bossard, della Società svizzera di radiologia medica, chiedeva che «il capitolo radiologia e tecniche di diagnostica per immagini, compresa la medicina nucleare [sia] esclusa dalla votazione odierna sulla struttura alfa 2.2». Il dott. Bossard spiega che non si tratta per i radiologi solamente della questione dell'apprezzamento delle prestazioni tecniche dal punto di vista economico, ma molto di più di questioni fondamentali, a fianco d'altre questioni di dettaglio. $\mathrm{Ci}$ sono quindi molte questioni aperte che sono appena evocate implicitamente, ma non per nome nella proposta del Comitato Centrale n. 1 ad tractandum 5, come aveva chiesto la società in occasione della Conferenza dei presidenti del 13 gennaio 2000. Il presidente $H$. H. Brunner è pronto a riprendere l'integralità del contenuto dei desideri della Società svizzera 
di radiologia medica nel pacchetto del round di revisione 2000, ricordando contemporaneamente che era stato il primo a indicare gli errori e le insufficiente denunciate dai radiologi e a finire per farlo ammettere agli assicuratori. Precisato ciò, per questioni formali, deve opporsi a questa proposta. Il dott. F. Bossard, rassicurato dalle garanzie ricevute, ritira la sua proposta.

Una proposta (n. 12 ad tractandum 5) del dott. $L$. Dubs, Società svizzera d'ortopedia, chiede che si esiga "che l'AM e l'AI si pronuncino sulla struttura alfa 2.2 prima che entri in vigore la tariffa AINF/AM/AI». Per utilizzare il gergo parlamentare, si tratta qui piuttosto di un'interpellanza che di una proposta, spiega il dott. H. H. Brunner. Segnala che la Commissione delle tariffe mediche LAINF (CTM) ha da parte sua accettato la struttura tariffaria in una seduta del dicembre 1999 e che si è ancora nel vago per quel che concerne la posizione definitiva dell'AM e dell'AI che, come si sa, agiscono nella sfera d'influenza del Dipartimento federale dell'interno. Questo deve ancora pronunciarsi. Se tarda a farlo, non si vorrà addossare la colpa del ritardo alla FMH ... Data quest'informazione non è più necessario votare su questa proposta.

La Camera si occupa in seguito della proposta $n$. 13 ad tractandum 5, presentata dal prof. T. Rüedi e dal dott. J. Ammann, della Società svizzera di chirurgia, e che tocca una questione fondamentale: "La struttura tariffaria non deve essere sottoposta al Consiglio federale per approvazione". A nome di chi ha presentato la proposta, l'avv. F. Bernath, segretario generale della FMS, fa presente le pressioni continue e costanti del capo del DFI per ottenere informazioni e documenti di tutti i generi all'attenzione del Consiglio federale, nella prospettiva di un'approvazione della struttura tariffaria da parte del governo. L'avv. Bernath ha effettuato uno studio approfondito della questione, per giungere alla conclusione che questa pretesa competenza del Consiglio federale per approvare la struttura tariffaria non esiste. Ci sarebbe competenza del Consiglio federale nel caso che esistesse una tariffa federale - che è una cosa diversa da una struttura tariffaria - o in caso di ricorso contro una decisione di un governo cantonale approvante una tariffa che concerne il settore della LAMal. L'avv. Bernath ha presentato il suo studio, per un "secondo parere», a tre famosi giuristi dell'ambiente universitario che, tutti e tre, hanno confermato la fondatezza delle sue conclusioni. Sotto questa luce, le pressioni incessanti esercitate dal DFI non hanno quindi ragione d'essere. Il presidente $H$. H. Brunner chiede una modifica di questa proposta. Spiega che abbiamo da tempo le stesse riserve dell'avv. Bernath (cfr. la pubblicazione dell'avv. H. P. Kuhn nel BMS n. 29/30/1999 - "Structures de tarifs, conventions tarifaires et neutralité des coûts dans la LAMal») e che abbiamo domandato a diverse riprese al DFI, sia verbalmente che per iscritto, di definirci questa procedura d'approvazione sulla forma e sul fondo. Sino ad oggi non abbiamo ricevuto nessuna risposta, cosa che non è necessariamente dovuta al caso ... Il presidente fa notare che ha anche sempre difeso questa posizione all'interno della TarMed, senza essere seguito.

Al termine della discussione, la Camera medica accetta la proposta della Società svizzera di chirurgia e la formula nel modo seguente: "Il Comitato Centrale è incaricato di esigere, sotto forma di ultimatum, le prese di posizioni necessarie e di rinunciare a presentare la struttura tariffaria al DFI e al Consiglio federale prima che questa situazione non sia stata chiarita". Questa proposta, accettata sotto questa forma dall'avv. Bernath a nome dei suoi mandanti, non deve impedire alla Camera medica di prendere oggi una decisione definitiva sulla struttura tariffaria alfa 2.2, dichiara il dott. H. H. Brunner come conclusione su questo punto all'ordine del giorno.

Il dott. Chr. Ramstein, presidente della Società di medicina del canton Soletta, presenta una proposta (n. 14 ad tractandum n. 5) in due punti, il primo dei quali riproduce perfettamente il testo della proposta del Comitato Centrale n. 1 ad tractandum 5, mentre il secondo intende ricordare che "la condizione essenziale ad un'applicazione della struttura tariffaria a livello svizzero non è solo la conclusione di un contratto quadro (convenzione tariffaria) tra la CTM/l'AI/ l'UFAM e la FMH, da una parte, e la CAMS e la FMH dall'altra, ma anche la conclusione di convenzioni tariffarie tra le federazioni cantonali degli assicuratori malattia e le società cantonali di medicina". Il presidente $H$. H. Brunner fa valere che l'essenziale di questa proposta è contenuta in quella (n. 1 ad tractandum n. 5) del Comitato Centrale e che si può quindi considerare integrata la proposta del dott. Ramstein; l'interessato dà il suo avvallo a questo modo di vedere la cosa, per cui non è necessario votare sulla sua proposta.

Conviene tornare sulle questioni di principio importanti sollevate dal $\mathrm{I}^{\circ}$ e dal $\mathrm{II}^{\circ}$ capoverso della proposta (n. 1 ad tractandum 3, 5 e 6) del dott. R. Streit, $B E$, (il $\mathrm{III}^{\circ}$ capoverso è già stato respinto all'inizio della seduta). In breve, il primo capoverso chiede che la struttura tariffaria alfa sia approvata "per un'introduzione a titolo di prova durante una fase pilota di un anno nel settore AINF/AM/AI" con un valore del punto di Fr. 1.-, e il secondo postula che ci si pronunci sull'adozione definitiva della struttura tariffaria, in particolare nel settore della LAMal, solamente una volta noti i risultati della fase pilota nel settore AINF/AM/AI. Il dott. Streit invita con determinazione la Camera a sostenere il suo punto di vista, se non vuole che il corpo medico "acquisti il gatto nel sacco" e sia definitivamente vincolata per il futuro; contesta che questo modo di fare equivalga ad un "no", cosa che - a suo parere - sarebbe un'interpretazione soggettiva. Non è il parere del dott. H. H. Brunner che ricorda che ci siamo creati la possibilità di disdire il contratto a breve termine. Il dott. J. Ammann, FMS, apporta il suo sostegno alla proposta del dott. Streit, perché senza votare "no" alla struttura tariffaria, desidererebbe poter beneficiare ancora di certe garanzie prima di un'accettazione definitiva. È la ragion per cui la FMS preconizza una votazione generale non immediatamente dopo la decisione della Camera 
medica, ma dopo un certo tempo d'osservazione dopo che questa struttura è entrata in vigore nella «Tariffa blu", dopo che l'idea di organizzarne una dopo un anno sia stata respinta (consultativamente) dalla Conferenza dei presidenti.

Quest'ultimo intervento porta il presidente a commentare le proposte del Comitato Centrale n. $1 \mathrm{ad}$ tractandum n. 1 e $n .1$ ad tractandum 5. Le proposte del Comitato Centrale rappresentano i suggerimenti essenziali in provenienza dalle diverse correnti all'interno della FMH (Modello FMS, revisione 2000) come anche le proposte della Camera concernente l'approvazione della struttura. Si noterà che il punto 4 della proposta n. 1 del Comitato Centrale ad tractandum n. 5 riprende, la proposta del dott. Ramstein, So, rinforzandola e mettendola su un terreno inattaccabile. Si aggiunge ora la questione della votazione generale: non sarebbe bene organizzarla immediatamente o rimandarla di un anno. Se il DFI vuole (o deve) veramente prendere una decisione, ciò dovrebbe avvenire in marzo o aprile, se si vuole che le casse malattia possano incominciare a calcolare le loro quote. Se le condizioni appena decise dalla Camera medica non potessero essere soddisfatte, la votazione generale sarebbe immediatamente bloccata. Questo modo di fare è comunicabile e ci garantisce il massimo di margine di manovra. Il presidente conclude invitando la Camera a respingere, per ragioni formali, la proposta del dott. R. Streit. Non viene più domandata la parola e si passa quindi alla votazione.

In una prima votazione la proposta del dott. $\mathrm{R}$. Streit è sottoposta alla Camera che la respinge con 129 voti contrari e 33 favorevoli, con qualche astensione.

In una seconda votazione la Camera è chiamata a pronunciarsi sulla questione della votazione a bollettini segreti che era stata chiesta da tre delegati. La votazione a bollettini segreti non ottiene il quarto dei voti come richiesto, poiché viene respinta con 129 voti contrari, 33 favorevoli e 6 astensioni.

In una terza votazione, la proposta del Comitato Centrale $n .1$ ad tractandum 5, il cui testo è riprodotto qui sotto in extenso nella prospettiva di una votazione generale, è accettato con 144 voti favorevoli, 30 contrari e 3 astensioni.

\section{Testo approvato dalla Camera medica} del 2 febbraio 2000

1. La Camera medica del 2 febbraio 2000 approva la versione alfa 2.2 della struttura tariffaria TarMed calcolata sulla base di un valore del punto tariffario di Fr. 1.-, e questo esclusivamente in vista della sua introduzione contrattuale nelle tariffe cantonali secondo la LAMal e nella tariffa AINF/AM/AI e nel catalogo delle prestazioni ospedaliere dagli assicuratori competenti e dalle organizzazioni di fornitori di prestazioni.

2. Il "Severity Complexity Score» fissato in modo generale a 1.2 per le prestazioni chirurgiche e intervenzionali è limitato a 2 anni al massimo. Questo deve essere sostituito da un nuovo modo di calcolo che si basa sul modello dei costi della FMS del 6 dicembre 1999. Le relative negoziazioni devono iniziare al più presto con gli assicuratori e terminare prima dell'entrata in vigore delle convenzioni tariffarie basate sulla struttura tariffaria TarMed.

3. La revisione 2000 costituisce parte integrante dell'approvazione della versione alfa 2.2 della struttura tariffaria; l'approvazione ha luogo con riserva del trattamento da parte degli assicuratori e dei fornitori di prestazioni, delle prestazioni e capitoli della banca dati della revisione 2000 (in particolare ginecologia/ostetricia e le tecniche di diagnostica per immagini).

4. L'introduzione contrattuale secondo la cifra 1 comprende, come condizione sine qua non, una convenzione tra i fornitori di prestazioni e gli assicuratori competenti concernente la neutralità dei costi in occasione dell'introduzione delle tariffe. Questa convenzione deve includere delle disposizioni obbligatorie allo scopo di ottenere, sul piano nazionale, una convergenza del valore del punto tariffario delle prestazioni mediche e delle prestazioni tecniche per il settore "studio medico" e "ospedale ambulatoriale" su una durata di 5 anni, in modo da raggiungere una varianza conforme alla gestione aziendale, ma di al massimo $+/-4 \%$ intorno ad un valore medio ponderato.

5. Il Comitato Centrale sottometterà entro la prossima seduta della Camera medica, ma al più tardi in occasione della seduta del giugno 2000, un rapporto e una proposta relativi ai punti 2 e 4.

Si giunge quindi alla questione della votazione generale a proposito della quale il segretario generale fornisce qualche indicazione d'ordine pratico. Il dott. L. Dubs, della Società svizzera d'ortopedia, ritira la sua proposta (n. 1 ad tractandum 5.2) facendo osservare che la sua accettazione ritarderebbe troppo la procedura. Viene fatta ancora qualche osservazione, in particolare da alcuni che desiderano che un certo tempo sia messo a disposizione per l'informazione della base o per essere fissato sulla portata di questa consultazione. Si vuole anche sapere di quale documentazione potranno disporre i nostri membri. Il presidente precisa agli uni che la votazione generale servirà a confermare $o$ invalidare la decisione che la Camera ha appena preso e che è da considerare come un pacchetto da approvare o rifiutare e ricorda agli altri che i corsi specifici sulla struttura alfa 2.2 sono già incominciati e proseguiranno e, cosa da non trascurare, che un'importante informazione è accessibile su "Internet" e lo sarà anche sotto forma di CD-Rom. L'alternativa "Gutenberg" pesa 7,5 chili ed è alta $62 \mathrm{~cm}$... Questa è la conseguenza di una tariffa estremamente differenziata che, non dimentichiamolo, è stata reclamata da diverse società specialistiche. Infine, in accordo con le società specialistiche, cercheremo di elaborare un estratto delle principali prestazioni frequentemente utilizzate dagli uni e dagli altri e, eventualmente, di pubblicarle nel BMS. Per tentare ancora una volta di dissipare i timori espressi vivamente dal dott. J. Ammann, FMS, il presidente fa ca- 
pire che la FMH, tramite la Camera medica, mantiene sempre la possibilità di riprendere la sua libertà, per esempio se le legittime domande dei ginecologi o dei radiologi fossero troppo trascurate. Riportiamo ancora l'intervento del dott. $R$. Urscheler, presidente della Società di medicina del canton San Gallo, che considera che la Camera non dovrebbe decidere di lanciare una votazione generale, ma deve lasciarne la responsabilità alle società che per statuto ne hanno la possibilità.

Si passa quindi alla votazione da cui risulta una maggioranza di 91 contro 54 e 10 astensioni per la votazione generale. Osservazione importante: per una malaugurata mancanza d'attenzione, il segretario generale aveva in un primo tempo annunciato alla Camera che si era pronunciata a favore della votazione generale. Si era trascurato il fatto che era necessaria una maggioranza dei due terzi. Una volta segnalato questo errore, qualche minuto più tardi, il risultato conforme alle esigenze statutarie è stato comunicato ai delegati: la maggioranza dei due terzi non era stata raggiunta.

\section{Convenzioni}

Ci si deve occupare ancora di due proposte dal contenuto quasi identico (proposte n. 1 e 2 ad tractandum 3.4, 3.5, 6 e 7) presentate, la prima dal dott. G.-P. Jenny, della Società svizzera di oftalmologia, e la seconda dal dott. L. Dubs, della Società svizzera d'ortopedia, che chiedono in sostanza di prevedere, in ogni convenzione, la possibilità per una società specialistica di disdire la convenzione TarMed per quello che la concerne. Il presidente $H$. H. Brunner fa osservare che, non fosse che per dei motivi formali, una società specialistica medica non potrebbe uscire da una convenzione tariffaria. Questo diritto appar- tiene alla FMH, al livello federale (Tariffa blu), e alle società cantonali o a dei medici individuali per le convenzioni tariffarie al livello cantonale. Conclusione: sulla base di queste spiegazioni oggettive, il dott. Jenny e il dott. Dubs non insistono più.

Restano infine sul tavolo presidenziale due proposte (n. 1 e 2 ad tractandum n. 6.1) depositate dal dott. W. Grete e M. F. Rübel, rispettivamente presidente e segretario della Società zurighese di medicina. Esse concernono tutte e due il testo ancora in progetto del contratto tra la FMH e gli assicuratori della Tariffa blu. Sarebbe prematuro trattarle qui, ma il dott. H. H. Brunner le prenderà in considerazione nelle negoziazioni future e ringrazia tutti coloro che gli hanno fatto pervenire dei suggerimenti.

Poiché si tratta del contratto con il Concordato, i negoziatori attendono ancore dalle società cantonali di medicina delle indicazioni chiare su quanto deve risaltare dalla tariffa quadro sul piano federale e quello che deve essere lasciato a livello cantonale.

$$
* * * *
$$

Il presidente uscente della Società medica della Svizzera romanda, il dott. A. E. Marmy, si congeda dalla Camera medica e coglie l'occasione per ringraziare, a nome di tutti, il presidente H. H. Brunner per l'enorme lavoro svolto (applausi nutriti). Il dott. H. H. Brunner si dice sensibile al complimento e riconoscente al suo autore. Dice di voler continuare a rimanere al servizio dell'insieme del corpo medico, ringrazia tutti i partecipanti alla presente seduta per la loro collaborazione costruttiva ed efficace e associa ai suoi ringraziamenti i collaboratori della segreteria generale, che si sono impegnati da mesi affinché questa seduta possa aver luogo (applausi).

La seduta è tolta. Sono le 17 e qualche minuto. 